\title{
EFFECT OF OCCLUSAL SCHEME ON BOLUS TRANSPORT DURING FEEDING FOR COMPLETE DENTURE WEARERS
}

\author{
Abdallah M. Ibrahim*
}

\begin{abstract}
Objectives: This study was aimed to evaluate the influence of different occlusal schemes on bolus movement during feeding for complete denture wearers.

Methods: Thirty completely edentulous patients were participated in this study. All patients were randomly classified in two equal groups. Group I received a complete denture with bilateral balanced occlusal scheme (BBO) and group II received a complete denture with lingualized balanced occlusal scheme (LBO). Patients were instructed to chew and swallow $1 / 4$ of cookie (coated with pudding and barium powder) with and without denture. Masticatory duration, number of chewing cycles, number of swallow, oropharyngeal residue, and penetration aspiration observation were measured by using videofluoroscopic assessment at baseline, 2 weeks and 3 months after denture insertion.
\end{abstract}

Results: Penetration of food into the larynx was significantly founded in edentulous patients without complete dentures. After 3 month of denture insertion, LBO group showed a statistically significant reduction in masticatory duration, and number of chewing cycles $(p<0.05)$ compared to BBO group.

Conclusion: The occlusal scheme has been found to be important in swallowing function as lingualized balanced occlusal scheme improve chewing ability and oral preparatory stage of swallowing in comparison to bilateral balanced occlusal scheme after 3 month of denture insertion.

KEYWORDS: occlusal scheme, complete denture, bolus, swallowing.

\section{INTRODUCTION}

Complete edentulism lead to weakening of the masticatory muscles and decrease the masticatory efficiency $^{(1)}$. Regaining the occlusal vertical dimension by wearing complete denture allows the recovery of oral anatomical structures and maintains pharyngeal anatomical morphology correctly ${ }^{(2)}$.
Properly designed conventional complete dentures also improve swallowing function in the oral phase (oral preparatory and oral transport) and pharyngeal phase of swallowing ${ }^{(3,4)}$. Proper contact between the tongue and the palatal surface of the denture improves bolus movements and decreases penetration of food into the larynx ${ }^{(5)}$.

\footnotetext{
* Lecturer of Removable Prosthodontics, Faculty of Dentistry, Mansoura University, Egypt.
} 
Several types of occlusal schemes were proposed for conventional complete denture occlusion such as, bilateral balanced occlusion, lingualized occlusion and monoplane occlusion. The ideal occlusion should allow the better masticatory efficiency through providing the highest forces of masticatory muscles during mastication ${ }^{(6)}$ while the number of contractions of the muscles was the least. ${ }^{(7)}$.

During solid food processing, the food was crushed into smaller particles and softened by chewing movement then the food was mixed with saliva to form a food bolus consistency which is ready for swallowing. ${ }^{(8)}$ It was reported that, masticatory duration needed to prepare the food particles to the proper size and consistency increased in complete denture wearers compared to dentate subjects because decreasing in masticatory efficiency ${ }^{(9)}$. These individuals reduce bolus consistency through long chewing time, subsequently, the bolus leading edge become deeper in the pharynx ${ }^{(10)}$, which increase the chance of laryngeal penetration or aspiration ${ }^{(11)}$.

Studies combining mastication and swallowing functions to investigate their interaction have been reported, among these studies the relation between mastication, swallowing and food bolus transport have been published ${ }^{(12,13)}$.

A study evaluated chewing solid bolus found that swallowing may be affected by the characters of masticatory apparatus, the tongue pressure and the movement of the head during swallowing ${ }^{(14)}$. Other studies showed a relation between masticatory duration and the function of swallowing found that the increased in the mastication time lead to the higher incidence of swallowing dysfunction and increasing the chance of aspiration ${ }^{(15)}$. On the other hand, The longer the presence of food bolus in the hypopharynx, the higher the risk of swallowing dysphagia $^{(16)}$.

According to our knowledge, the literature lacks studies on the influence of the denture occlusion on bolus transport during feeding for complete denture patients. Thus, the aim of the present study was to verify the influence of different occlusal schemes on food bolus transport during feeding for patients wearing conventional complete denture.

\section{MATERIALS AND METHODS}

Thirty completely edentulous patients were participated in this study (18 men and 12 women) their ages range from 50- 70 years and the mean age was 60 years. The participants presented to Prosthodontics Department, Faculty of Dentistry, Mansoura University for complete denture treatment. All participants became completely edentulous for at least 6 months and have no previous experience with removable prosthesis. Subjects with temporomandibular joint disorders, sleep disorders, head/ neck injury, or with skeletal deformity were excluded from this study. All participants had Angel's class I maxillo-mandibular relation. Informed consent was written from each patient, and approval from the Faculty Ethical Committee was obtained.

\section{I - Prosthetic Procedures:}

1. Preliminary impressions were made using irreversible hydrocolloid impression material (CA37. Superior pink. Cavex. Holland Bv) for fabrication of diagnostic casts. Custom trays were fabricated using auto-polymerizing resin (Acroston, cold curing acrylic resin, Egypt). The Border was molded with low fusing impression compound sticks then the final impressions were made by using zinc oxide non-eugenol impression material (Cavex impression past, Holland). Final impressions were poured using hard dental stone.

2. After construction of upper and lower record blocks, it's contour, height and orientation were adjusted. The upper cast was mounted on a Whip-Mix semi-adjustable articulator (Courtesy of the Whip-Mix, Corp. USA) using a face-bow record (fig.1). Centric inter-occlusal records were registered. These records were obtained for mounting the mandibular cast. 


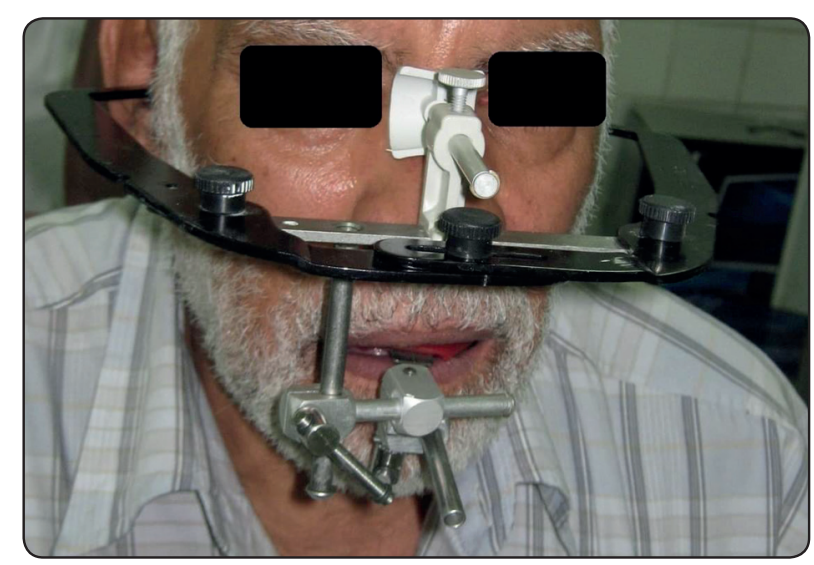

Fig. (1) Face bow record

3. Dentures were constructed with two posterior occlusal schemes using semi-anatomic artificial teeth (Naperce, Yamahachi Dental MFG.CO. Japan):

- Bilateral balanced occlusal scheme: The anterior teeth were set according to esthetic and phonetic requirement. Protrusive inter- occlusal wax wafer record was obtained to adjusting the horizontal condylar guidance and the lateral condylar guidance was adjusted according to Hanaue equation. The posterior teeth were arranged as the palatal cusps of the upper posterior teeth were set in the central groove of the lower posterior teeth and the upper buccal cusps were kept in contact with lower buccal cusps. During lateral and protrusive movement, bilateral posterior and anterior contact were present and the buccal cusps and palatal cusps kept in articulation and functional.

- Lingualized balanced occlusal scheme: the upper buccal cusps were raised so no contact between the upper buccal cusps and the lower buccal cusps in both centric and eccentric movements.

4. The participants were randomly classified into two equal groups, 15 patients in each group.

Group I (BBO): Patients received the denture with Bilateral balanced occlusal scheme.
Group II (LBO): Patients received complete denture with Lingualized balanced occlusal scheme.

5. Occlusal adjustment was carried out by using laboratory remount after deflasking. Using two colored articulating paper the maximum intercuspation, as well as lateral excursive and protrusive movements were refined by selective and spot grinding then the teeth were polished.

6. The dentures were delivered to the patient and then clinical remount were done for both groups. The occlusal adjustement was done by selective and spot grinding and follow-up appointments were scheduled.

\section{II- Evaluation method:}

Each patient was subjected to videofluoroscopic imaging 3 times, without denture (T0), after 2 weeks (T1), and after 3 months (T2) of denture delivery.

\section{Videofluroscopic (VF) assessment:}

All participants were evaluated by using videofluoroscopic evaluation of bolus movement. Each patient was asked to chew $1 / 4$ of cookie (coated with pudding and barium powder) and swallow the bolus once they were ready ${ }^{(17)}$. The videofluoroscopic recordings were then transferred to the computer for later data analysis. EO program (Version 1.36) was used to analyzed the videofluoroscopic recordings by placing numbers (1/100 second) on the video frame for later frame-by-frame data analysis.

\section{Swallowing evaluation involve the following pa- rameters}

- Masticatory duration: Started from once the patient masticate the food bite to the start of the movement of the food bolus posteriorly. Number of chewing cycles: Up-down movement of the jaw until beginning of swallowing.

- Number of swallow: swallows required to empty a bolus from the oral cavity. 
- Oro-pharyngeal residue: A scale was used to detect the amount of food residue in the oral cavity, the valleculae, and the posterior pharyngeal wall $(0=$ no residue, $1=$ coating, $2=$ pooling) .

Penetration/Aspiration observation: P\A Scale $(0=$ "no penetration," 1 = "pentration," $2=$ "aspiration") The patients were evaluated for penetration and aspiration of bolus. If the bolus enter the airway down to the level of the vocal folds it mean penetration while below the level of the vocal folds it mean aspiration.

\section{Statistical analysis:}

Recorded data was used for comparison between groups. Wilcoxon signed rank test was used to compare quantitative data. While Mc Nemar test was used to compare qualitative data. Significance was considered if $\mathrm{P}$ value was less than 0.05. Statistical analysis software (SPSS Statistics version 17) was used to perform the statistical analyses.

\section{RESULTS}

The means for masticatory duration (in seconds), number of chewing cycles and number of swallows for $\mathrm{BBO}$ and $\mathrm{LBO}$ groups were presented in table (1) and table (2) respectively. The results of this study showed that, for both groups there was a statistically significant reduction of masticatory duration and number of chewing cycles $(\mathrm{p}<0.05)$ after denture insertion $(\mathrm{T} 1, \mathrm{~T} 2)$ compared to edentulous state(T0) and also a statistically significant reduction of masticatory duration and number of chewing cycle at $\mathrm{T} 2$ when compared to $\mathrm{T} 1 \quad(\mathrm{p}<0.05)$.

All recordings included more than 2 swallow. For both groups, there was a statistically significant reduction $(\mathrm{p}<0.05)$ for number of swallow at $\mathrm{T} 2$ evaluation period compared to both $\mathrm{T} 1$ and edentulous state, while non significant difference $(p>0.05)$ was present between edentulous state and $\mathrm{T} 1$ evaluation period .

When comparing between $\mathrm{BBO}$ and $\mathrm{LBO}$ groups There was a statistically non significant difference for masticatory duration, chewing cycles , and number of swallows at $\mathrm{T} 0$ and $\mathrm{T} 1$. While at $\mathrm{T} 2$ evaluation period, LBO group showed a statistically significant reduction in masticatory duration, and number of chewing cycles $(p<0.05)$ and nonsignificant difference for number of swallows .

Regarding to Oro-pharyngeal residue, a coating was founded at edentulous state and at $\mathrm{T} 1$ evaluation period in all patients (fig.2) while at T2 there was no residue for both groups.

Penetration/aspiration observation showed no aspiration was observed while, penetration was presented in edentulous state for most of patients (12 patients for BBO group and 13 patients for LBO group) (fig. 3) while significant absence of penetration was found after denture insertion in both groups.

TABLE (1): The means of masticatory duration, chewing cycles, and number of swallows for BBO group.

\begin{tabular}{|c|c|c|c|c|c|c|}
\hline & T0 & T1 & $\mathbf{T} 2$ & \multicolumn{3}{|c|}{$P$ value } \\
\hline & Mean \pm SD & Mean \pm SD & Mean \pm SD & T0T1 & T0T2 & T1T2 \\
\hline Masticatory duration & $23.2 \pm 3.1$ & $11.1 \pm 2.1$ & $9.5 \pm 1.7$ & .002 & .001 & .004 \\
\hline Chewing cycles & $22.6 \pm 4.1$ & $15.8 \pm 3.3$ & $13.8+3.1$ & .004 & .002 & .002 \\
\hline No. of swallows & $3.2 \pm 0.7$ & $3.1 \pm 0.5$ & $2.30 .4 \pm$ & 0.4 & .001 & .001 \\
\hline
\end{tabular}

Wilcoxon signed rank test 
TABLE(2): The means of masticatory duration, chewing cycles, and number of swallows for LBO group.

\begin{tabular}{|c|c|c|c|c|c|c|}
\hline & T0 & T1 & T2 & \multicolumn{4}{|c|}{ Pvalue } \\
\cline { 2 - 7 } & Mean \pm SD & Mean \pm SD & Mean \pm SD & T0T1 & T0T2 & T1T2 \\
\hline Masticatory duration & $23.5 \pm 2.1$ & $10.2 \pm 2.1$ & $8.2 \pm 1.3$ & 0.002 & 0.001 & 0.002 \\
\hline chewing cycles & $23 \pm 3.5$ & $15.7 \pm 2.5$ & $13.7 \pm 2.5$ & 0.002 & 0.001 & 0.01 \\
\hline No. of swallows & $3.4 \pm 0.6$ & $3.3 \pm 0.7$ & $2.5 \pm 0.5$ & 0.3 & 0.001 & 0.002 \\
\hline
\end{tabular}

Wilcoxon signed rank test

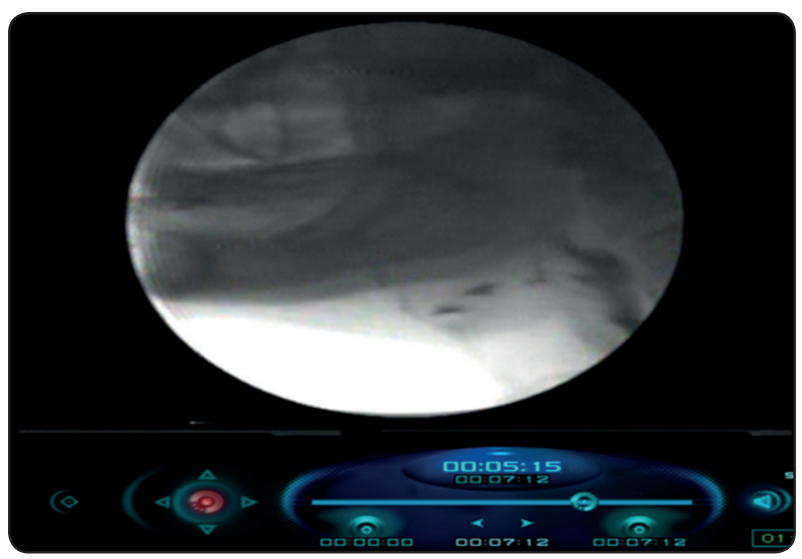

Fig. (2) Oropharyngeal residue

\section{DISCUSSION}

Wearing conventional complete denture drastically change the occlusal contact and the shape of the oral cavity. During swallowing. The occlusal contact provide an anchorage to the mandible which contribute to a sequential coordination among masticatory muscles ${ }^{(18)}$. A video-fluoroscopy study found that complete loss of teeth made it difficult for older adults to chew and swallow ${ }^{(4)}$. In this study, the masticatory duration and number of chewing cycles were found to be increased in edentulous state than after denture insertion. The cause of this change might be due to manipulation of food bolus in the oral cavity without denture was difficult as the complete denture work with the tongue, cheeks, and mandible during chewing ${ }^{(19)}$ to form well-formed and lubricated food bolus for smooth swallowing ${ }^{(20)}$. When the patients not wearing the denture, the food was dispersed in the oral cavity as

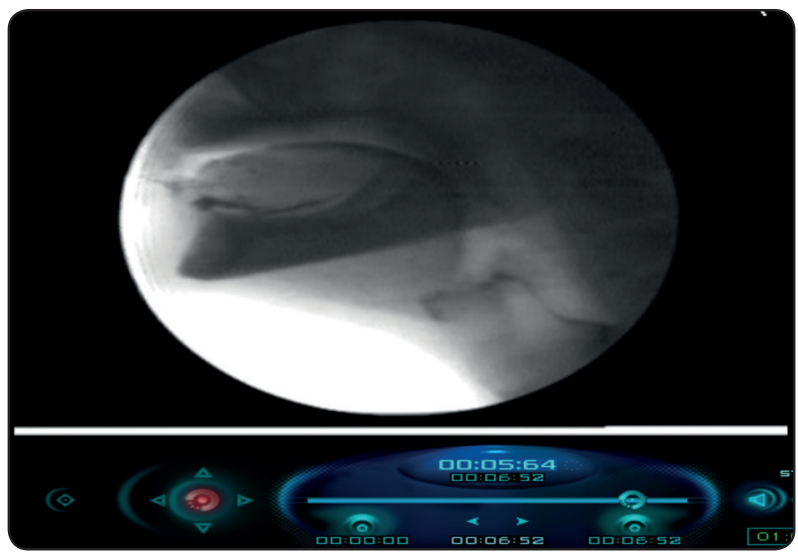

Fig. (3) Laryngeal penetration

the patients were less able to aggregate and collect the food into bolus. This result was consistent with previous study which found that without complete denture the duration of oral preparatory stage of swallowing was increased ${ }^{(13)}$.

In this study all patients swallow more than 2 swallow until finishing the bolus. Commonly, When ingesting hard food, several swallows were found even for one bite. Each swallow had a portion of the food, and the remaining food was swallowed with subsequent swallow. Okada et al. study found that when eating food freely, humans need more than one swallows, even with a single bite of food, although the first swallow include the most of the food. (21)

Ligualized balanced occlusal scheme showed a lower masticatory duration, and lower chewing cycles than bilalteral balanced occlusal scheme. It may be due to lingualized occlusal schemde offered 
a higher ability of food crushing, displayed faster as well as smoother chewing movement compared with bilateral balanced occlusal scheme suggesting good bolus formation and transportation during feeding. This was in accordance with Deniz and Ozkan study which founded that lingualized balanced occlusal scheme produced a higher activity of masticatory muscles and a lower masticatory duration than bilateral balanced occlusal scheme, ${ }^{(22)}$ Also, Lemos et al ${ }^{(23)}$ founded that lingualized occlusal scheme had better masticatory performance index as it needed lower occlusal force to increase masticatory performance than other occlusal schemes so it considered as the prefered oclusal scheme.

It has been reported that the intereference of coordinated swallowing movments lead to pentration of food into the larynx ${ }^{(24)}$. In this study, Laryngeal penetration was presented in patients not wearing the dentures than after denture wearing, this may be due to loss of occlusal support in edentulous patient lead to deterioration of the coordinated swallowing movements between the hyoid, the larynx and the tongue. This result was coincide with Yoshikawa et al. study which found that penetration of food into the larynx was higher in frequency in completely edentulous patients not wearing the dentures ${ }^{(3)}$.

The results of this study reported a significant reduction for masticatory duration, chewing cycles, number of swallows and oropharyngeal residue after 3 months of denture insertion. This may be due to neuromuscular adaptation to the complete denture as the chewing process is a neuromuscular function including speed, effective movements and continuous masticatory forces..$^{(22,25,26)}$

In this study, there was non-significant differences was present between $\mathrm{BBO}$ and $\mathrm{LBO}$ groups after 2 week of denture insertion and a significant differences was found after 3 months of dentur insertion. This may be due re- establishment of chewing ability for newly inserted denture need a period of 3 months after the dentures insertion. ${ }^{(27)}$ So, evaluating the effect of occlusal schemes should be done after at least 3 months of occlusal adjustment session.

\section{CONCLUSION}

Oral rehabilitation of edentoulous patients with conventional complete dentures is still the protective treatment that restor the chewing ability and swallowing function to avoid the risk of laryngeal penetration during feeding.

The occlusal scheme has been found to be important in swallowing function as lingualized balanced occlusal scheme improve chewing ability and oral preparatory stage of swallowing in comparison to bilateral balanced occlusal scheme after 3 month of denture insertion.

\section{REFERENCES}

1. Moradpoor H, Salari F, Ebadian B, Raissi S, Shirani M.(2018). Patient satisfaction with occlusal scheme of conventional complete dentures: A randomised clinical trial (Part II). J Oral Rehabil., 45:702-709

2. Prachi Singhal, Sameer Singhal, Abinav Dagar.(2018) Impact of dentures wearing on retropharyneal space in edentulous patients; data from rural tertiary hospital. International Journal of Contemporary Medical Research, 5(4):D37-D40.

3. Yoshikawa M, Yoshida M, Nagasaki T, Tanimoto K, Tsuga K, Akagawa Y. (2006). Influence of aging and denture use on liquid swallowing in healthy dentulous and edentulous older people. J Am Geriatr Soc., 54: 444-9.

4. Yoshikawa M, Yoshida M, Nagasakin T, Tanimoto K, Tsuga K, Akagawa Y.(2008). Effects of tooth loss and denture wear on tongue-tip motion in elderly dentulous and edentulous people. J Oral Rehabil., 35: 882-8.

5. Furuya J, Tamada Y, Sato T, Hara A, Nomura T, Kobayashi $\mathrm{T}$ et al. (2015). Wearing complete dentures is associated with changes in the three-dimensional shape of the oropharynx in edentulous older people that affect swallowing. Gerodontology, 33: 513-521.

6. Zarb GA, Hobkirk J, Eckert S, Jacob R.(2013). Prosthodontic Treatment for Edentulous Patients-E-Book: Complete Dentures and Implant- Supported Prostheses: Elsevier Health Sciences (ed 12). St. Louis: Mosby.

7. Moradpoor H, Arabzade Hoseini M, Savabi O, Shirani M.(2017). Patient satisfaction with occlusal scheme of conventional complete dentures: A randomised clinical trial (part I). J Oral Rehabil.,45:41-49. 
8. Woda A, Mishellany A, Peyron MA.(2006). The regulation of masticatory function and food bolus formation. J Oral Rehabil., 33:840-849.

9. Veyrune JL, Lassauzay C, Nicolas E, Peyron MA, Woda A.(2007). Mastication of model products in complete denture wearers. Arch Oral Biol.,52:1180-1185.

10. Matsuo K, Kawase S, Wakimoto N, Iwatani K, Masuda Y, Ogasawara T. (2013). Effect of viscosity on food transport and swallow initiation during eating of two-phase food in normal young adults: a pilot study. Dysphagia.,28:63-68.

11. Saitoh E, Shibata S, Matsuo K, Baba M, Fujii W, Palmer JB.(2007) Chewing and food consistency: effects on bolus transport and swallow initiation. Dysphagia., 22:204-209.

12. Sato T, Furuya J, Tamada Y, Kondo H. (2013). Impacts of palatal coverage on bolus formation during mastication and swallowing and subsequent adaptive changes. J Oral Rehabil.,40:751-757.

13. Yamamoto H, Furuya J , Tamada Y,\& H. Kondo H. (2013). Impacts of wearing complete dentures on bolus transport during feeding in elderly edentulous. J Oral Rehabil., 40: 923-931.

14. Takeda S, Saito E, Matsuo K, Baba M, Fujii W, Palmer JB.(2002). Influence of chewing on food transport and swallowing. Jpn J Rehabil Med.39:322-330.

15. Yoshida FS, Mituuti CT, Totta T, Berretin-Felix G. (2015). Influence of the masticatory function on the swallowing in the healthy elderly. Audiol Commun Res., 20(2):161-6

16. Iizumi T, Yoshino M, Kagaya H, Hori K, Ono T. (2018). Effect of tongue-palate contact mode on food transport during mastication. J Oral Rehabil., 45:605-611.

17. Abou-Elsaad T (2003). Effect of diet variables on the biomechanical measures of swallowing in stroke patients. International congress series journal, 1240c: 683-688.
18. Kondoh. J, Ono T, Tamine K, Fujiwara S, Minagi Y,Hori K, Maeda Y, Kreissl M and Nitschke I. (2015). Effect of complete denture wearing on tongue motor biomechanics during swallowing in edentulous older adults. Geriatr Gerontol Int., 15: 565-571

19. Mioche L, Hiiemae KM, Palmer JB. (2002). A postero-anterior videofluorographic study of the intra-oral management of food in man. Arch Oral Biol.,47:267-280.

20. Matsumaru Y. (2010). Influence of mandibular residual ridge resorption on objective masticatory measures of lingualized and fully bilateral balanced denture articulation. J Prosthodont Res., 54:112-118.

21. Okada A, Honma M, Nomura S, Yamada Y. (2007). Oral behavior from food intake until terminal swallow. Physiol Behav., 90: 172-179.

22. Deniz DA, Kulak Ozkan Y. (2013). The influence of occlusion on masticatory performance and satisfaction in complete denture wearers. J Oral Rehabil., 40:91-98.

23. Lemos CAA, Verri FR, Gomes JML, Santiago Júnior JF, Moraes SLD, Pellizzer EP.(2018). Bilateral balanced occlusion compared to other occlusal schemes in complete dentures: A systematic review. J Oral Rehabil., 45:344-354.

24. Logemann JA. (1998). Evaluation and Treatment of Swallowing Disorders, 2nd Ed. Austin, TX: Pro Ed,

25. Hatch JP, Shinkai RS, Sakai S, Rugh JD, Paunovich ED. (2001). Determinants of masticatory performance in dentate adults. Arch Oral Biol., 46:641-648.

26. Galo R, Vitti M, Mattos Mda G, Regalo SC. (2007). Masticatory muscular activation in elderly individuals during chewing. Gerodontology., 24:244-248.

27. Farias-Neto A, Carreiro AF. (2015). Changes in patient satisfaction and masticatory efficiency during adaptation to new dentures. Compend Contin Educ Dent., 36:174-177. 